\title{
Suitability of blue filter ophthalmic lenses with respect to the use with mobile devices and computers
}

\section{Idoneidad de las lentes oftálmicas con filtro azul respecto al uso de dispositivos móviles y ordenadores}

\author{
J. Arines ${ }^{1, S^{*}}, 0$. Rodríguez \\ 1.Departamento de Física Aplicada, Facultade de Óptica e Optometría, Universidade de Santiago de \\ Compostela \\ (*)E-mail: justo.arines@usc.es \\ S: miembro de SEDOPTICA / SEDOPTICA member \\ Received: 04/10/2020 Accepted: 01/02/2021 \\ DOI: $10.7149 /$ OPA.54.1.51048
}

\begin{abstract}
:
Blue light comprises the electromagnetic spectrum between approximately 380-500 $\mathrm{nm}$. The sun is the main source of blue light, although we also receive emissions of this type of light by electronic devices and artificial lighting. Changes in lighting systems and lifestyle habits have led to the questioning of exposure to blue light emitted by artificial sources as a possible health problem. Faced with this potential problem, ophthalmic lens laboratories brought to market lenses with specific treatment against hazard blue light emitted by electronic devices. In this work we measure the spectral transmittance of 6 blue filter ophthalmic lenses from 4 different laboratories, Essilor, Hoya, Shamir and Kodak. We face the transmission spectra measured with the emission spectra of 3 different electronic devices, to be able to discuss the suitability of these blue filter lenses for use with electronic devices. We conclude that the transmittance of blue filters in the maximum blue light emission range is high above $90 \%$, and that changing the correlated color temperature of electronic devices results in a greater reduction in exposure to hazard blue light. We also found that Shamir's Blue Zero and Essilor's Eye Protect System lenses block UVA light and have low transmittance in the range of 380$420 \mathrm{~nm}$, so they would be more effective for protection against blue light outdoors than for use with electronic devices.
\end{abstract}

\section{Key words:}

\section{RESUMEN:}

La luz azul comprende el espectro electromagnético visible entre los 380-500 nm aproximadamente. El sol es la principal fuente de luz azul, aunque también recibimos emisiones de este tipo de luz por parte de los dispositivos electrónicos y la iluminación artificial. Los cambios en los sistemas de iluminación y los hábitos de vida han provocado el cuestionamiento de la exposición a la luz azul emitida por las fuentes artificiales como un posible problema de salud. Ante esta posible problemática los laboratorios de lentes oftálmicas sacaron al mercado lentes con tratamiento específico frente a la luz azul nociva emitida por los dispositivos electrónicos. En este trabajo medimos la transmitancia espectral de 6 lentes oftálmicas con filtro azul procedentes de 4 laboratorios distintos, Essilor, Hoya, Shamir y Kodak. Enfrentamos los espectros de transmisión medidos con los espectros de emisión de 3 dispositivos electrónicos distintos, con el fin de poder discutir la idoneidad de estas lentes con filtro azul para su uso con dispositivos electrónicos. Concluimos que la transmitancia de los filtros azules en el rango de máxima emisión de luz azul es alta, superior al 90\%, y que modificando la temperatura de color correlacionada de los dispositivos electrónicos se consigue una mayor reducción en la exposición a la luz azul nociva. Así mismo comprobamos que las lentes Blue Zero de Shamir y Eye Protect System de Essilor bloquean la luz UVA y presentan una transmitancia baja en el rango de 380-420 nm, por lo que serían más eficaces para la protección frente a la luz azul en exteriores que para el uso con dispositivos electrónicos.

Palabras clave: Filtro azul, Luz azul nociva, Transmitancia. 


\section{REFERENCES AND LINKS / REFERENCIAS Y ENLACES}

[1] G. Wyszecki, W.S. Stiles, Color Science: Concepts and methods, quantitative data and formulae. New York: Wiley, (1982).

[2] M.G. Figueiro,, L. Sahin, M. Kalsher, B. Plitnick, M.S. Rea, "Long-Term, All-Day Exposure to CircadianEffective Light Improves Sleep, Mood, and Behavior in Persons with Dementia", Journal of Alzheimer's Disease Reports, 4(1), 297-312 (2020)

[3] J. Zhou, JR Sparrow, "Light filtering in a retinal pigment epithelial cell culture model." Optom Vis Sci, 88, 759-65 (2011)

[4] JJ. Hunter, JI. Morgan, WH. Merigan et al. "The susceptibility of the retina to photochemical damage from visible light". Prog Retin Eye Res. 31(1), 28-42 (2012)

[5] J.G. Lawrenson, C.C. Hull, L.E. Downie, "The effect of blue-light blocking spectacle lenses on visual performance, macular health and the sleep-wake cycle: a systematic review of the literature". Ophthalmic and Physiological Optics, 37(6), 644-654 (2017).

[6] S. Laxhmi Chellappa et al. "Non-Visual Effects of Light on Melatonin, Alertness and Cognitive Performance: Can Blue-Enriched Light Keep Us Alert?", PloS one, 6(1), e16429 (2011).

[7] E. Sánchez, Hicimos la luz y perdimos la noche, Universidad de Cantabria, (2017).

[8] Essilor España [Internet], 2016, Crizal Prevencia las lentes inteligentes- Essilor. [Consultado en julio 2019] Disponible en: https://essilor.es > Productos (2019)

[9] Essilor España, [Internet], 2016, Eye Protect System: lentes transparentes para proteger de la luz nociva, [Consultado en julio 2019] Disponible en: https://essilor.es > Productos (2019)

[10] Shamir Optycal Industry Ltd, [Internet], 2019, Materiales Shamir Spain, [Consultado en julio 2019]

[11] Hoya Vision Care, [Internet] 2019, Para estilos de vida digitales-Hoya Vision Care, Consultado en julio 2019, Disponible en: https://www.hoyavision.com/es/verproductos/profesionales/tratamientos-antirreflejantes/de-vida-digitales/ (2019)

[12] Kodak lens, [Internet, 2019] Protección contra la luz azul: KODAK Lens BluProtect UV. [Consultado en julio 2019] Disponible en: https://kodaklens.es/productos/tratamientos/blueprotectproteccion-luz-azul/ (2019)

[13] C. Fernández-Vigo, M. Argilés, E. Pérez, G. Cardona, "Radiancia espectral de lentes oftálmicas con filtros de luz azul", Optica Pura y Aplicada, 50(2), 165-172 (2017)

[14] L. E. Downie, "Blue-light filtering ophthalmic lenses: to prescribe, or not to prescribe?" Ophthalmic and Physiological Optics, 37(6), 640-643 (2017).

[15] R. Nagare, B. Plitnick, MG. Figueiro, "Does the iPad Night Shift mode reduce melatonin suppression?" Light Res Technol., 51(3), 373-383 (2019).

[16] JA. Calvo-Sanz, CE. Tapia-Ayuga, "Blue light emission spectra of popular mobile devices: The extent of user protection against melatonin suppression by built-in screen technology and light filtering software systems", Chronobiol Int. 37(7), 1016-1022 (2020).

[17] R. Martín Catalán, "Caracterización espectrofotométrica de lentes de contacto y de lentes oftálmicas", Trabajo de Fin de Grado en Óptica y Optometría, Dirigido por Ana Sánchez-Cano, Universidad de Zaragoza (2018).

[18] M. Spitschan, R. Lazar, C. Cajochen, "Visual and non-visual properties of filters manipulating shortwavelength light", Ophthalmic and Physiological Optics, 39(6), 459-468 (2019).

[19] T. W. Leung, R.W.H. Li, C.S. Kee, "Blue-light filtering spectacle lenses: optical and clinical performances". PloS one, 12(1), e0169114(2017).

[20] https://fluxometer.com/rainbow/\#!id=Outdoors/10AM\%20LA\%20sunny

[21] https://fluxometer.com/rainbow/\#!id=iPad\%20Pro/5000K-iPad\%20Pro 


\section{Introduction}

La luz azul comprende la región del espectro electromagnético visible entre 380-500 nm [1]. Se sabe que la luz azul es beneficiosa para la salud, influye en el funcionamiento del ritmo circadiano, en el estado de ánimo y concentración.[2] La principal fuente de luz azul es el sol. Hoy en día la mayoría de las fuentes de luz artificial que utilizamos, pantallas de televisión, móviles, tablets, ordenadores, luces LED, etc, emiten grandes cantidades de luz azul. Sin embargo, esta emisión es muy inferior a la que proporciona el sol. En la figura 1 mostramos los espectros de emisión del sol a las 10.00 horas un día soleado y de una lámpara LED blanco frio $5000 \mathrm{~K}$. En la figura también se observa la curva de sensibilidad a la luz azul nociva (Blue light Hazard IC62471). La zona blanquecina es la resultante de multiplicar el espectro de luz por la curva de sensibilidad a la luz azul nociva. Estas figuras se extrajeron del enlace indicado en el pie de figura.

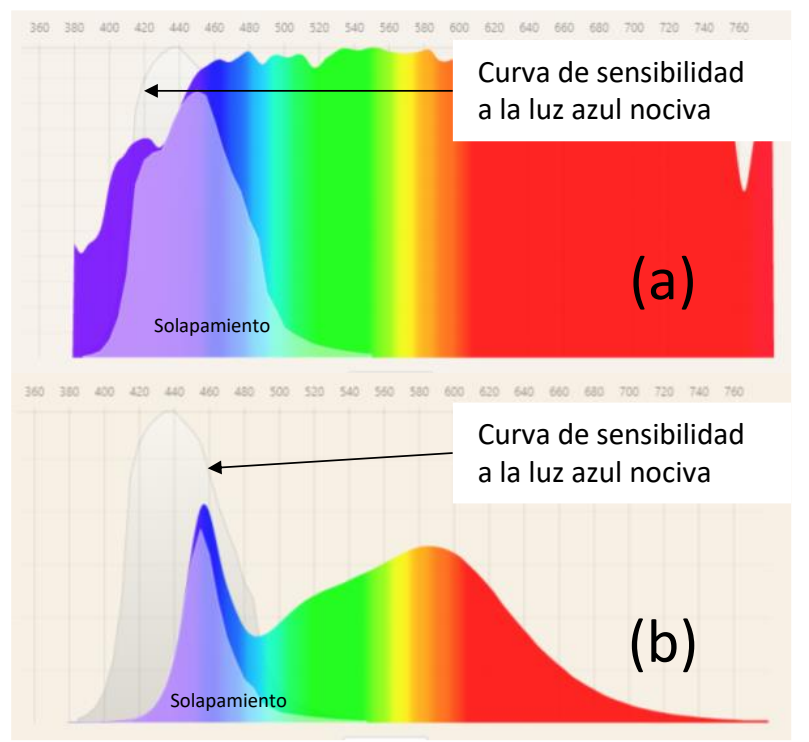

Figura 1: Espectros de emisión del sol a las 10.00 horas un día soleado (izquierda) y de un LED blanco frio (derecha). Imágenes extraídas de la página https://fluxometer.com/rainbow/\#!id=Outdoors/10AM\%20LA\%20sunny\&action=BlueLightHazard.

Se sabe que si comparamos la cantidad de luz azul que reciben los ojos en un minuto al aire libre frente a la recibida de los dispositivos electrónicos y luces LED, al aire libre recibimos mucha más cantidad. Sin embargo, hoy en día pasamos la mayor parte del día en espacios interiores bajo luz artificial. Existen estudios que relacionan la exposición prolongada a la luz azul que emiten las pantallas de visualización con fototoxicidad a nivel retiniano, así como cambios en el ritmo circadiano. [3-5] El daño retiniano fototóxico depende de la intensidad de la luz, de la longitud de onda y del tiempo de exposición. La exposición prolongada a longitudes de onda corta (entorno a $440 \mathrm{~nm}$ ) podría producir (en caso de superar la dosis umbral) daño fotoquímico por su absorción por las células del epitelio pigmentario retiniano y fotorreceptores, desencadenando un proceso oxidativo que a largo plazo podría provocar la muerte celular, llegando a desarrollar enfermedades retinianas como la degeneración macular asociada a la edad [3-5].

Por otra parte, la exposición prolongada a la luz azul emitida por los dispositivos electrónicos y fuentes LED, produce alteraciones en el ritmo circadiano [5-7]. El ritmo circadiano es el reloj interno que regula las funciones fisiológicas del organismo, manteniendo el buen funcionamiento de los órganos vitales, sistema endocrino, hormonal, etc. La hormona más importante que regula el sistema circadiano es la melatonina, sintetizada en la glándula pineal. Las células ganglionares intrínsecamente fotosensibles de la retina (ipRGC) que absorben la luz azul y contienen melanopsina están conectadas con la glándula pineal (donde se sintetiza la melatonina). La absorción de luz azul en el entorno de los $490 \mathrm{~nm}$ provoca la inhibición de la liberación de la melatonina, de forma que en una situación normal, en la que la exposición a la luz azul desaparecería al llegar la noche, esta hormona se empezaría a liberar. Sin embargo, nuestras noches no son normales, en cuanto a que por la iluminación de nuestras casas, oficinas y pantallas seguimos expuestos a la luz azul produciéndose una inhibición de la liberación de melatonina y por tanto una alteración de nuestro ritmo circadiano, provocando problemas de sueño, y cambios fisiológicos que derivan en la aparición de enfermedades [7]. 
La curva de sensibilidad a la luz azul nociva se encuentra entre los 380 y los $550 \mathrm{~nm}$, alcanzando su máximo entorno a los $440 \mathrm{~nm}$. Por otra parte, la curva de visión fotópica estaría comprendida entre 420 y $680 \mathrm{~nm}$ con su pico máximo en $555 \mathrm{~nm}$. Mientras que la curva de sensibilidad melanópica abarca el rango de $400 \mathrm{a}$ $590 \mathrm{~nm}$ con su pico máxima en $490 \mathrm{~nm}$.

En los últimos años han aparecido tres soluciones al problema de la exposición inadecuada a la luz azul: 1) filtros que se colocan sobre las pantallas de visualización; 2) lentes oftálmicas con filtro de luz azul incorporado; 3) programas informáticos que permiten modificar la temperatura de color correlacionada (TCC) de la pantalla de visualización. En cuanto a las lentes oftálmicas con filtro azul (objeto de este trabajo) nos podemos encontrar con lentes que incorporan el filtro a modo de tratamiento superficial, o lentes que incorporan el filtro en el propio material del que están hechas. Las lentes con filtro azul se ofertan al consumidor para que proteja sus ojos frente a la luz azul emitida por las pantallas y dispositivos electrónicos. Únicamente se comenta su utilidad en este ámbito, en ningún momento se hace referencia a su posible capacidad protectora frente a la luz azul procedente de lámparas LED o del propio sol, dando a entender que los esfuerzos comerciales se centran en atender a la sensación de riesgo que se ha creado respecto al uso de los dispositivos electrónicos. En las especificaciones de las lentes, los fabricantes describen el producto enfocándolo a prevenir los problemas visuales desencadenados por la luz azul nociva que emiten las pantallas de visualización como: cansancio ocular, picor y enrojecimiento, insomnio, riesgo de degeneración macular asociada a la edad. En las referencias [8-12] recogemos los panfletos publicitarios de las distintas lentes empleadas en el presente trabajo

En un trabajo previo C. Fernández et. al. [13] analizaron la respuesta espectral de un conjunto de lentes oftálmicas que presentaban filtro azul. Comprobaron que las lentes proporcionaban distinto porcentaje de absorción en el pico de emisión de la Tablet que emplearon en el estudio, con un máximo de $17 \%$ y un mínimo de $2.9 \%$. En ese mismo año se publicó un artículo editorial en el que se debatía la idoneidad de prescribir o no las lentes con filtro azul. En él se sugería la necesidad de aumentar la evidencia científica sobre su eficacia [14]. En el trabajo de Nagane et. al. [15] se muestra la reducción en la inhibición de melatonina gracias a usar el modo nocturno en el Ipad, e incluso como el reducir la TCC del dispositivo aún acentúa más el efecto. Recientemente Calvo-Sanz et. al. [16] mostraron una comparativa del índice de supresión melanópica asociada a un gran número de dispositivos móviles en los que se empleó software de control de emisión de luz azul, llegando a la conclusión de que el efecto protector del uso de software de control de luz azul respecto a no usar el software es reducido.

El objetivo de nuestro trabajo es ir un poco más allá del realizado por C. Fernández et. al. al comparar el espectro de la radiación que llega al ojo al observar la fuente de luz a través de lentes con filtro azul o modificando la TCC de la fuente. Pretendemos además valorar la idoneidad de las lentes oftálmicas con filtro azul para la reducción a la exposición frente a la luz azul nociva y luz azul en el rango melanópico, emitida por los dispositivos electrónicos. En las siguientes secciones presentaremos el material y la metodología empleada, y los resultados. Seguiremos con la discusión de los resultados y terminaremos con las conclusiones.

\section{Materiales y métodos}

Empleamos 7 tipos de lentes distintas de 5 fabricantes diferentes. Seis de estas lentes presentaron filtro azul, mientras que la séptima fue una lente convencional con antirreflejante verde. En la tabla 1 presentamos los nombres comerciales de las lentes con filtro azul, nombre del fabricante, porcentaje de bloqueo de luz azul y rango del espectro indicado por el fabricante.

TABLA 1: Datos de bloqueo proporcionado por los fabricantes

\begin{tabular}{|c|c|c|c|}
\hline \hline Fabricante & Tratamiento & $\%$ bloqueo azul & $\begin{array}{c}\text { Rango espectro de } \\
\text { bloqueo }\end{array}$ \\
\hline \hline ESSILOR & PREVENCIA & $20 \%$ & \\
ESSILOR & EYE PROTECT SYSTEM & $20 \%$ & $420-520 \mathrm{~nm}$ \\
HOYA & BLUE CONTROL & $25 \%$ & \\
KODAK & BLUE PROTECT UV & & \\
KODAK & POWER KODAK & $10 \%$ & $450 \mathrm{~nm}$ \\
SHAMIR & BLUE ZERO & \multicolumn{2}{|l}{} \\
\hline
\end{tabular}


Para medir la transmitancia de las distintas lentes empleamos el espectrorradiómetro Blue-Wave (StellarNet) que proporciona medidas radiométricas en el rango de $350 \mathrm{~nm}$ a $1100 \mathrm{~nm}$ con una resolución de $2 \mathrm{~nm}$. Como fuente de luz empleamos una lámpara halógena. En la figura 2 mostramos el montaje experimental empleado. En la esquina superior derecha de la imagen, se observa la disposición de la fuente de luz, la lente y la sonda del espectrorradiómetro.

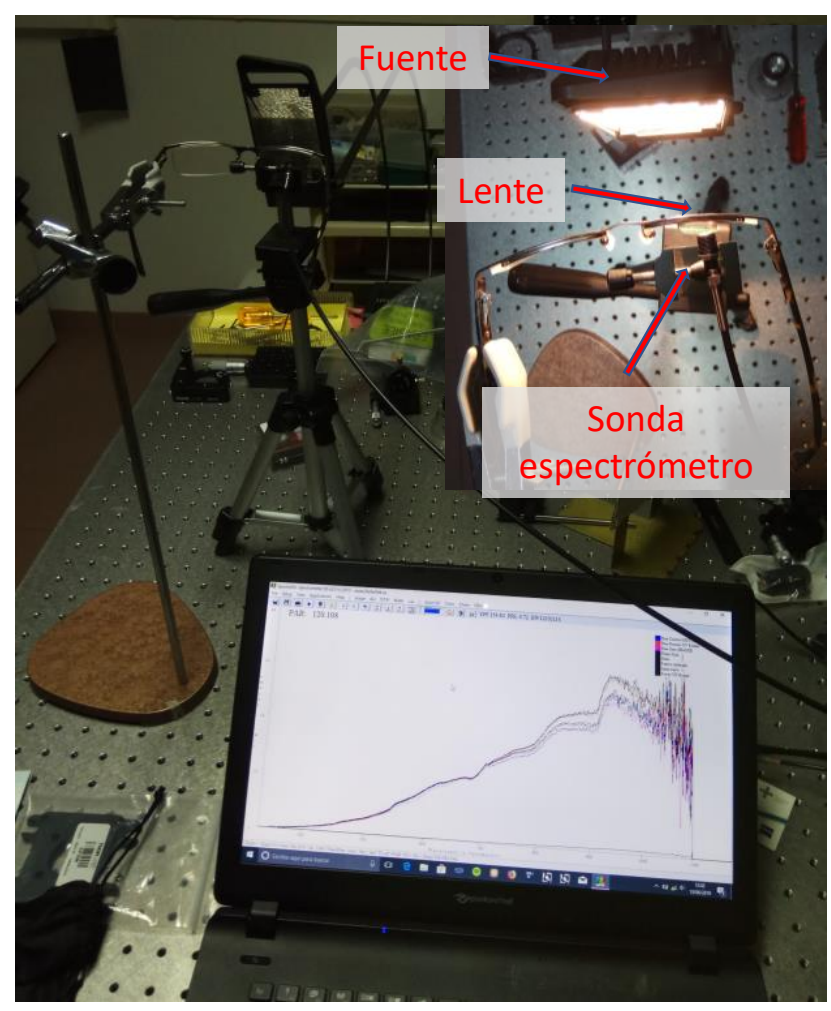

Figura 2: montaje experimental

La transmitancia ( $T)$ se midió de la siguiente forma: 1) se midió el espectro de la fuente $\left.\left(I_{0}\right) ; 2\right)$ Colocamos la lente a medir en el soporte; 3) Se midió el espectro transmitido (I);4) Se repitió el proceso para las 6 lentes; 5) Se calculó la transmitancia para cada una de las lentes empleando la siguiente expresión $T=I / I 0$. La distancia entre la sonda, la lente a medir y la fuente se mantuvo constante durante todas las medidas.

Los espectros de emisión de los dispositivos electrónicos con los que se trabajó se obtuvieron de la página web https://fluxometer.com/. En esta página web se pueden encontrar un gran número de espectros de emisión de distintas lámparas comerciales y dispositivos móviles operados a diferentes temperaturas de color. El uso de esta web facilitó la obtención de los espectros de emisión de los dispositivos, así como el poder haber trabajado con los espectros seleccionados (cuyos dispositivos asociados no se encontraban disponibles en el laboratorio). En la figura 3 exponemos los espectros (para dos TCC distintas, $5000 \mathrm{~K}$ y $2700 \mathrm{~K}$ ) correspondientes a los siguientes dispositivos: IPAD PRO; Galaxy S3; y Mac Book Air. La curva blanquecina se corresponde con la curva de sensibilidad de la luz azul nociva. 


\section{Espectros de emisión de los dispositivos estudiados}
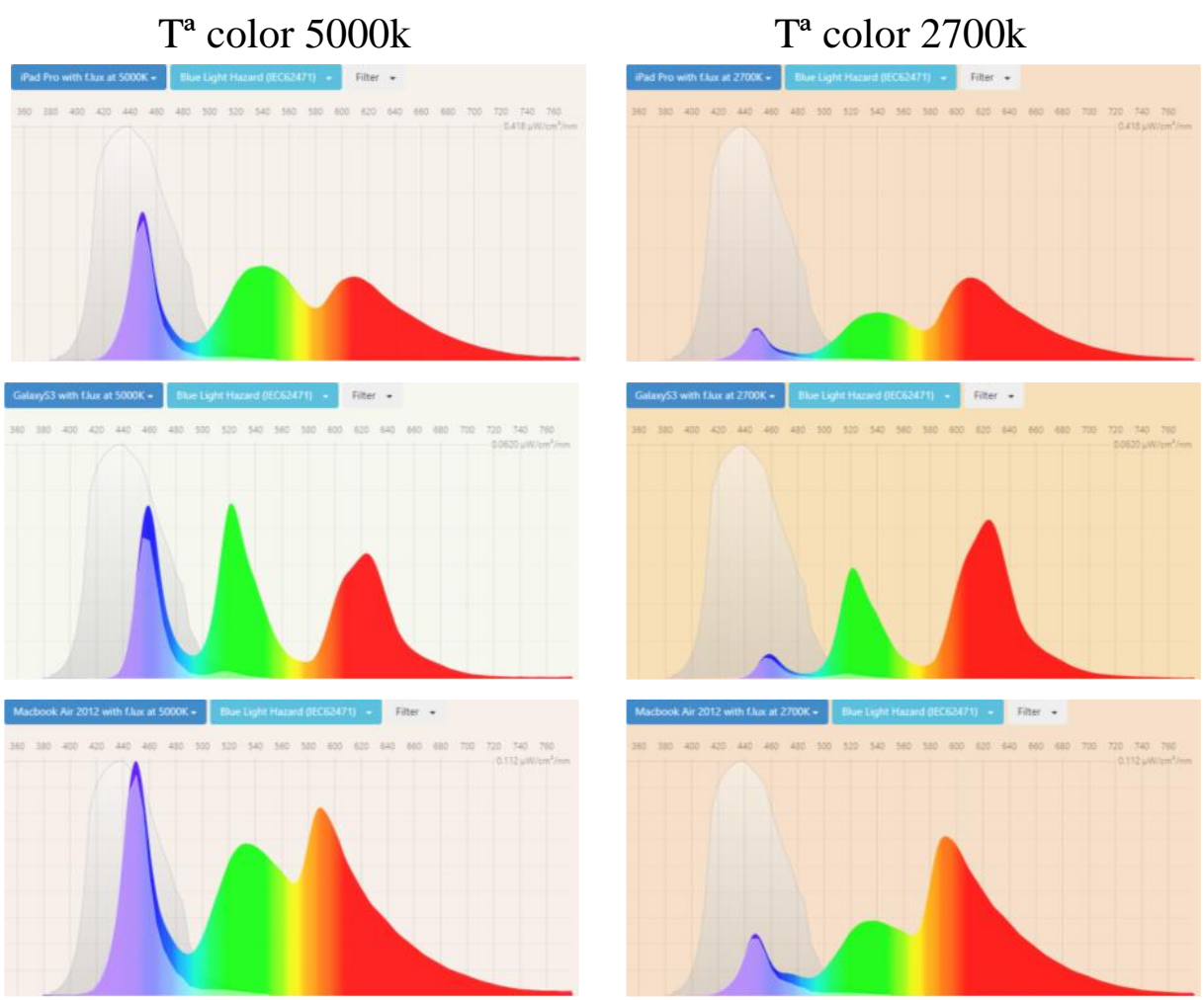

Figura 3: Espectros de emisión de distintos dispositivos para dos temperaturas de color distintas.

Podemos observar en la columna de la izquierda de la figura 3 (TCC de $5000 \mathrm{~K}$ ) que los dispositivos contemplados en el estudio presentan una emisión alta en el rango de los azules. Mientras que al bajar la TCC a $2700 \mathrm{~K}$ (columna de la derecha) la emisión este rango, disminuye significativamente.

\section{Resultados}

Empezamos esta sección presentando el espectro medido de la fuente (figura 4). Escogimos una fuente halógena en vez de una fuente LED por su mayor emisión en longitudes de onda cortas.

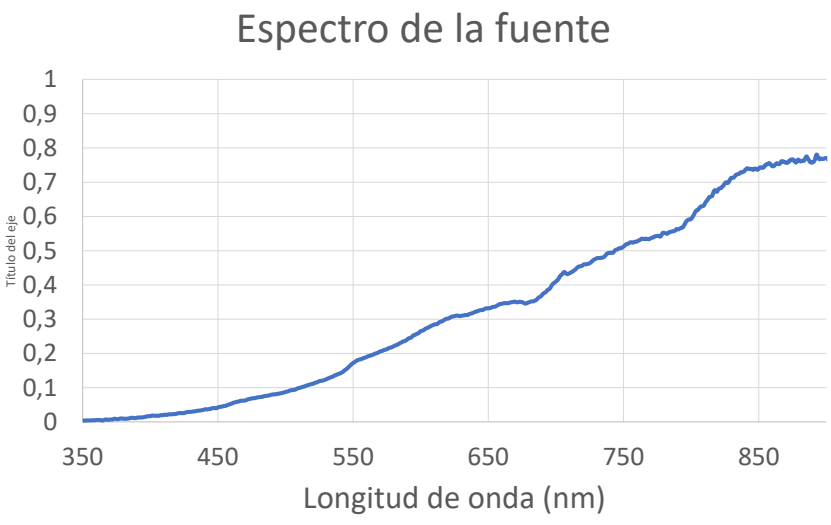

Figura 4: Espectro de la fuente

En la figura 5 presentamos las transmitancias de las distintas lentes analizadas: azul (Power Kodak), azul claro (Blue Zero de Shamir), verde (Blue Protect UV de Kodak), azul marino (Blue Control de Hoya), vermello (Eye protect System de Essilor), azul marino (Blue Control de Hoya), gris (Crizal Prevencia,de essilor) y rojo (lente convencional Sin filtro Azul y antirreflejante verde). A primera vista se observa una gran diferencia en el corte de longitudes de onda en el rango de 350-410 nm. Las lentes con tratamiento Eye 
Protect System y Blue Zero, destacan por bloquear la transmisión de luz en este rango, mientras que las otras lentes presentan un comportamiento muy semejante, permitiendo el paso de estas longitudes de onda. La inestabilidad de las líneas en las longitudes de onda entre $350 \mathrm{~nm}$ y $420 \mathrm{~nm}$ se debe a la baja relación señal de las medidas en este rango debido a que la fuente emite poca luz en estas longitudes de onda. Este problema hubiera sido mayor si hubiéramos usado como fuente una lámpara LED que presenta una emisión incluso menor en este rango. Así mismo en el caso de haber usado la fuente LED, la inestabilidad también se habría encontrado en el rango de longitudes de onda próximas al infrarrojo.

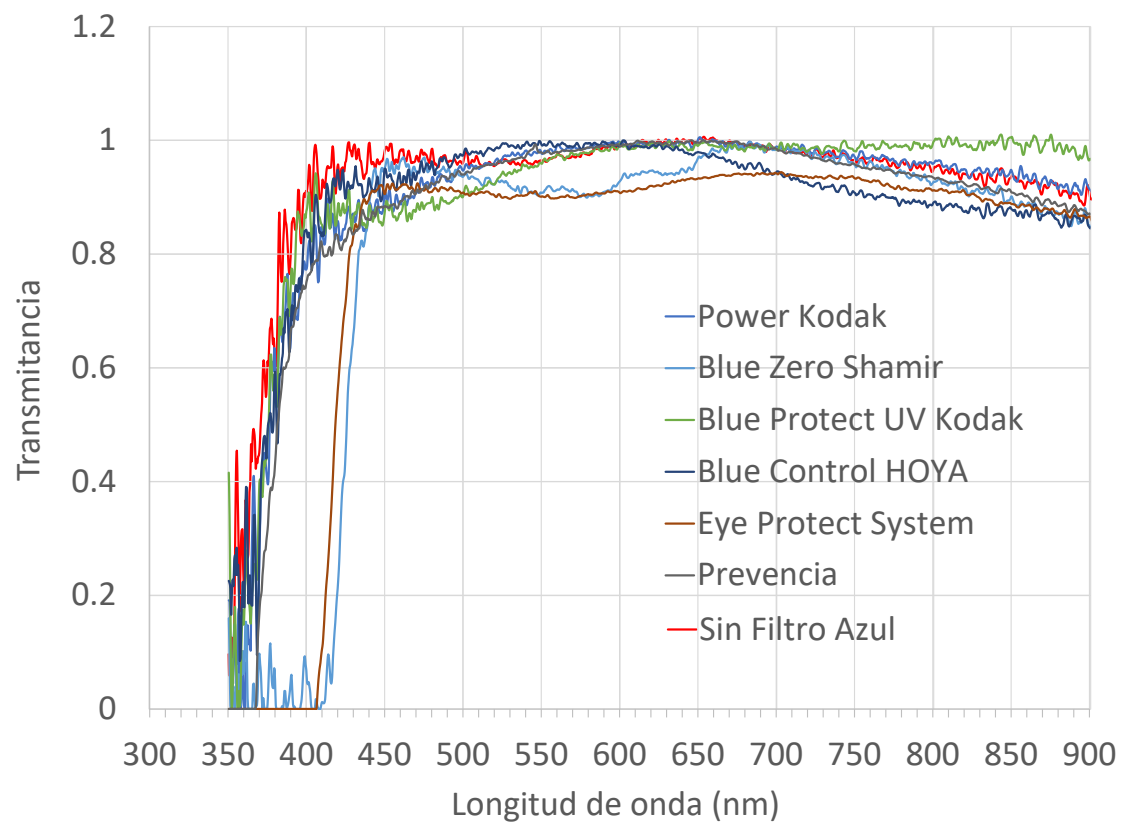

Figura 5: Transmitancias de las distintas lentes analizadas: azul (Power Kodak), azul claro (Blue Zero de Shamir), verde (Blue Protect UV de Kodak), azul marino (Blue Control de Hoya), vermello (Eye protect System de Essilor), azul marino (Blue Control de Hoya), gris (Crizal Prevencia,de essilor), y rojo (lente convencional sin filtro azul y antirreflejante verde).

En la figura 6 comparamos ahora la transmitancia de las lentes oftálmicas con las curvas de sensibilidad espectral de la luz azul nociva (línea gruesa azul), la luz azul melanópica (línea gruesa verde) y la curva de visión fotópica (línea gruesa gris).

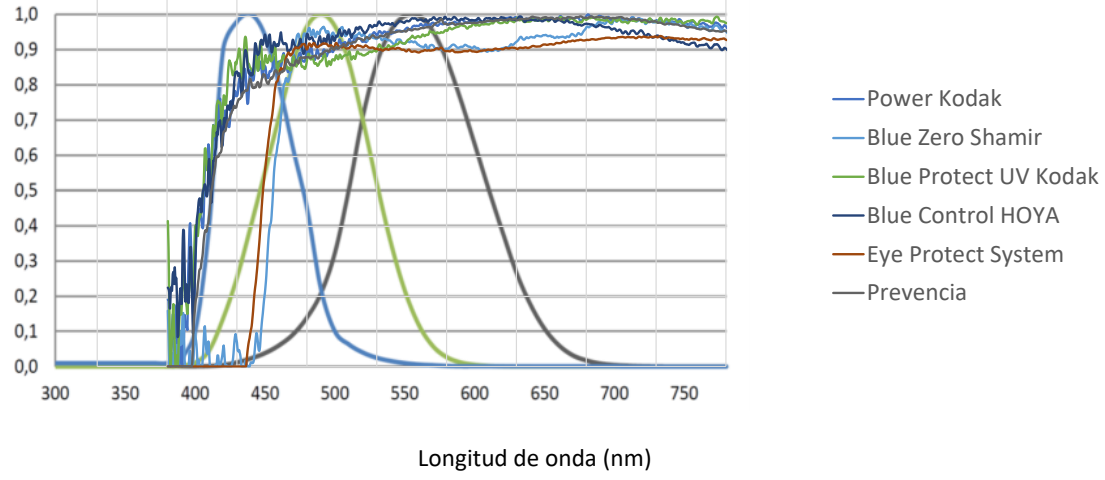

Figura 6: Comparativa de la transmitancia de las lentes con las curvas de sensibilidad a la luz azul nociva (línea azul), curva melanópica (línea verde), curva de visión fotópica (línea gris).

Tal como se recoge en la figura 6, las lentes con filtro azul cortan un porcentaje muy bajo de luz azul nociva, a excepción de las lentes Eye Protect System y Blue Zero que bloquean el espectro totalmente entre $350 \mathrm{~nm}$ y $410 \mathrm{~nm}$. En la tabla 2 presentamos la transmitancia media en \%, en los rangos (380-410) nm y (410-550) $\mathrm{nm}$, ambos rangos comprendidos dentro de la curva asociada a la luz azul nociva. También incluimos el rango (420 - 520) nm que es el rango empleado por los fabricantes para presentar sus datos de bloqueo. 
TABLA 2: Transmitancia media por rango de longitudes de onda (en \%)

\begin{tabular}{|l|c|c|c|c|c|c|c|}
\hline \hline $\begin{array}{l}\text { Rango de } \\
\text { longitudes } \\
\text { de onda (nm) }\end{array}$ & $\begin{array}{c}\text { Power } \\
\text { Kodak }\end{array}$ & $\begin{array}{c}\text { Blue Zero } \\
\text { Shamir }\end{array}$ & $\begin{array}{c}\text { Blue Protect } \\
\text { UV Kodak }\end{array}$ & $\begin{array}{c}\text { Blue Control } \\
\text { HOYA }\end{array}$ & $\begin{array}{c}\text { Eye Protect } \\
\text { System }\end{array}$ & $\begin{array}{c}\text { Prevencia } \\
\text { Sin filtro } \\
\text { Azul }\end{array}$ \\
\hline \hline $350-410$ & 73 & 2 & 79 & 73 & 0 & 68 & 85 \\
$410-550$ & 92 & 83 & 90 & 95 & 85 & 91 & 96 \\
$420-520$ & 91 & 87 & 89 & 95 & 89 & 90 & 97 \\
\hline \hline
\end{tabular}

En cuanto a la transmitancia en el rango de la curva de sensibilidad melanópica ocurre algo parecido. Las lentes con menor transmitancia son Eye Protect System y Blue Zero, que transmiten un 90\% dentro de ese rango. El resto de las lentes analizadas presentan una transmitancia aún mayor.

Vamos a comparar ahora la transmitancia de las lentes con el espectro de emisión de las fuentes seleccionadas, para dos TCC distintas (5000 K y $2700 \mathrm{~K})$. En la figura 7 presentamos las gráficas que permiten la comparación.
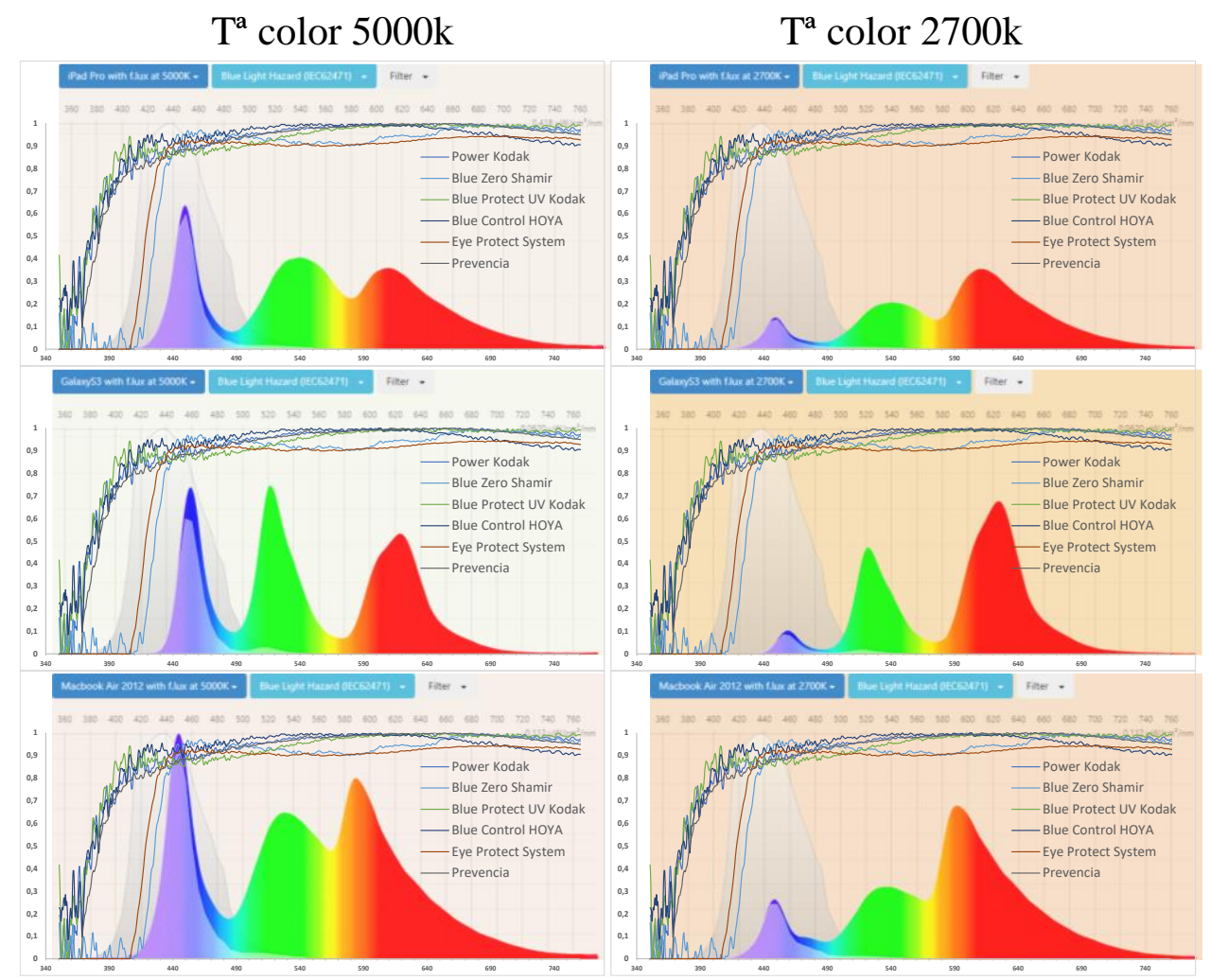

Figura 7: Transmitancias, y espectros de emisión de IPAD PRO, Galaxy S3 y Macbook Air, para dos TCC (5000 Ke 2700 K) y curvas de sensibilidad de luz azul nociva.

Se puede observar en la figura 7, en la región de la luz azul nociva, que la emisión es muy semejante en todos los dispositivos. El pico máximo de emisión en esta región se encuentra en torno a 450 - $460 \mathrm{~nm}$. En este rango de longitudes de onda, todas las lentes analizadas presentan una transmitancia en torno al 90\%. Como indicamos anteriormente, las lentes que menor transmitancia presentan en la región de luz azul nociva son las lentes Eye Protect System de Essilor y la Blue Zero de Shamir. Estas lentes bloquean la emisión de la luz azul comprendida entre los $380 \mathrm{~nm}$ y los $410 \mathrm{~nm}$, justo donde la emisión de la luz azul por las pantallas es mínima (como se observa en la figura 7). Por otra parte, si comparamos los espectros de emisión correspondientes a las dos TCC (5000 K y $2700 \mathrm{~K}$ ) comprobamos la gran diferencia existente entre ellos para todos los dispositivos.

Hemos visto la gran transmitancia de las lentes en la región del espectro donde los dispositivos electrónicos emiten la luz azul nociva (del orden del 90\%). Vamos ahora a ver cómo es la transmitancia en el rango de la 
luz azul melanópica. En la figura 8 presentamos las curvas de transmitancia, junto con los espectros de emisión de los distintos dispositivos y la curva de sensibilidad melanópica. Se observa que la curva de sensibilidad melanópica se encuentra en el rango del espectro visible comprendido entre $420-580 \mathrm{~nm}$. En esta región las 6 lentes estudiadas tienen un comportamiento muy parecido, transmitiendo un $90 \%$ de la luz en este rango. Si bajamos la TCC de 5000 K a 2700 K, vemos que la emisión de luz azul en esta región disminuye significativamente.

\section{$\mathrm{T}^{\mathrm{a}}$ color $5000 \mathrm{k}$}

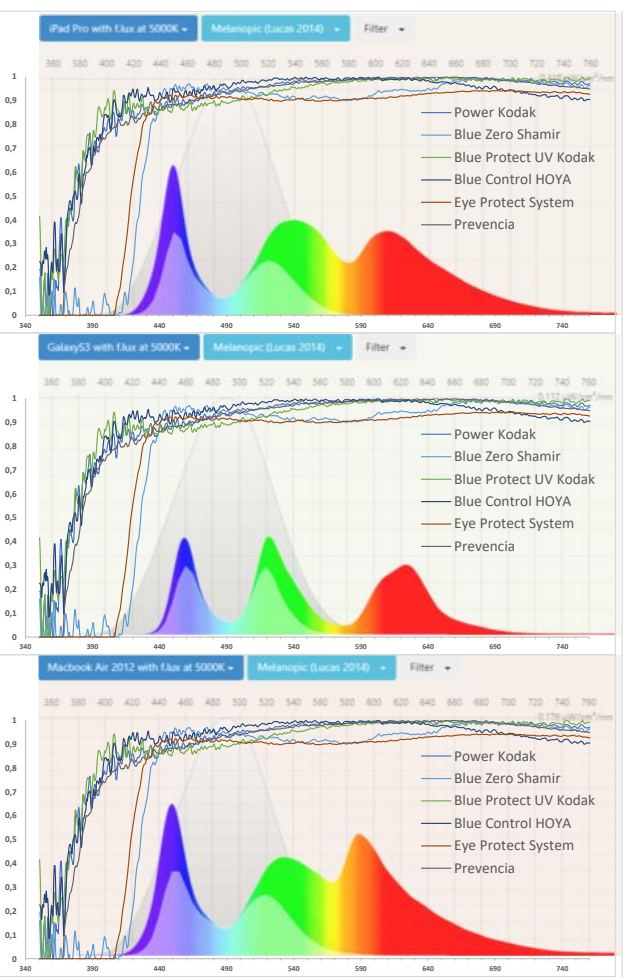

$\mathrm{T}^{\mathrm{a}}$ color $2700 \mathrm{k}$

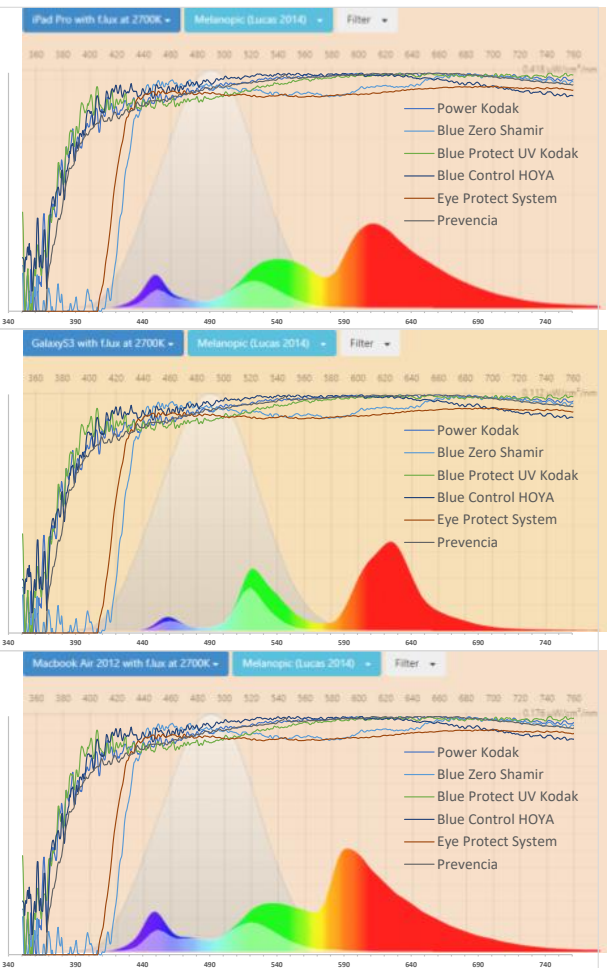

Figura 8: Transmitancias, espectro de emisión del IPAD PRO, Galaxy S3 y Macbook Air, para dos TCC (5000 K e 2700 K), y curvas de sensibilidad melanópica.

Por otra parte, se calculó el área bajo la curva de la función resultante de multiplicar la curva de sensibilidad a la luz azul nociva por el espectro de emisión de la fuente obteniéndose la irradiancia ponderada proporcionada por el dispositivo. Si además multiplicamos por el tiempo, tendremos la dosis acumulada. En la tabla 2 recogemos las dosis alcanzadas durante una hora de exposición a cada uno de los dispositivos contemplados en el estudio, en el rango de la luz azul nociva, y rango melanópico.

TABLA 3: Dosis de Luz Azul nociva y luz azul melanópica, en una hora de exposición $\left(\mathrm{mJ} / \mathrm{cm}^{2}\right)$

\begin{tabular}{|c|c|c|c|}
\hline Dosis de Luz Azul nociva & Ipad Pro & Galaxy S3 & MacAirBook 2012 \\
\hline Distancia de Trabajo & $15 ”$ & $9 "$ & $19 \%$ \\
\hline Filtro Azul (90\% transmitancia) y dispositivo con $\mathrm{T}^{\mathrm{a}} 5000 \mathrm{~K}$ & 21.42 & 2.98 & 9.99 \\
\hline $\mathrm{T}^{\mathrm{a}} 5000 \mathrm{~K}$ & 23.8 & 3.33 & 11.10 \\
\hline $\mathrm{T}^{\mathrm{a}} 2700 \mathrm{~K}$ & 5.67 & 0.58 & 3.14 \\
\hline Dosis de Luz Azul melanópica & Ipad Pro & Galaxy S3 & MacAirBook 2012 \\
\hline Distancia de Trabajo & $15 ”$ & $9 "$ & $19 \%$ \\
\hline Filtro Azul (90\% transmitancia) y dispositivo con $\mathrm{T}^{\mathrm{a}} 5000 \mathrm{~K}$ & 0.29 & 0.05 & 0.13 \\
\hline $\mathrm{T}^{\mathrm{a}} 5000 \mathrm{~K}$ & 0.32 & 0.06 & 0.15 \\
\hline $\mathrm{T}^{\mathrm{a}} 2700 \mathrm{~K}$ & 0.12 & 0.02 & 0.06 \\
\hline
\end{tabular}

Cada dispositivo se consideró que se encontraba a una distancia típica de trabajo. Los datos fueron extraídos del conjunto de datos que proporciona la página web https://fluxometer.com/. En la tabla 2 se puede 
apreciar que para cada uno de los dispositivos, la reducción que se obtiene en la dosis, es mucho mayor si se reduce la TCC del dispositivo que usando las lentes con filtro azul.

\section{Discusión}

Parte importante de este trabajo es aportar información útil para dar respuesta a las siguientes preguntas ¿Compro las lentes para mis gafas con el filtro azul?; ¿Debo vender las lentes con el filtro azul?; ¿La protección que ofrecen compensa el precio del tratamiento? Estas son preguntas que tanto usuarios como trabajadores de establecimiento de óptica se hacen a la hora de comprar o vender unas gafas. Tanto vendedores como compradores saben que existen los tratamientos y filtros frente a la luz azul, tanto por la propaganda que se puede encontrar en los medios de comunicación como por los documentos técnicos que los fabricantes proporcionan a los establecimientos de óptica. Todas las lentes que analizamos en este trabajo están recomendadas por sus fabricantes por sus efectos protectores frente a la luz azul emitida por las pantallas de los dispositivos electrónicos, a excepción de la lente que no presenta filtro azul.

Los resultados obtenidos muestran que la transmitancia de las lentes analizadas en el rango de la luz azul nociva es muy alta, en general superior al 90\%. Lo mismo sucede en el rango de sensibilidad melanópico. Por otra parte, hemos mostrado que la disminución de la TCC de las pantallas de visualización de $5000 \mathrm{Ka}$ $2700 \mathrm{~K}$ supone una reducción notable de la emisión de luz azul nociva y luz azul melanópica. Por ello ponemos en duda que la recomendación que se deba realizar para reducir la exposición a la luz azul nociva procedente de dispositivos móviles sea usar lentes oftálmicas con filtro azul, siendo más adecuado o idóneo, disminuir la TCC y brillo de estos. Con este simple cambio, se observa que llegaría al ojo menor cantidad de luz azul nociva o luz azul melanópica que empleando cualquiera de las lentes analizadas en este trabajo.

Nuestros hallazgos corroboran los obtenidos en otros estudios [13, 16-19]. En estos trabajos se muestra la alta transmitancia de los filtros azules de las lentes oftálmicas y su reducido efecto protector. A diferencia con estos trabajos, nosotros analizamos el efecto de los filtros considerando el espectro de emisión de un conjunto de pantallas de visualización mostrando que, en el rango de emisión de estas fuentes, las curvas de transmitancia son casi planas con una transmitancia próxima al 90\%. Así mismo, en este trabajo hemos medido la transmitancia de una lente convencional sin filtro azul, y con antirreflejante verde. En la tabla 2 se recogen los valores medios de transmitancia en los rangos de longitudes de onda analizados. Se puede comprobar que transmite un $6 \%$ más que el promedio de las transmitancias de las lentes que presentan filtro azul. Únicamente un 6\% más de transmisión.

El hecho de determinar las curvas de transmitancia nos permite conocer cómo será el espectro de luz que llega al ojo, una vez escogido el dispositivo de iluminación. Si hubiéramos escogido otra metodología, por ejemplo, empleando un conjunto limitado de dispositivos móviles que estuvieran a nuestra disposición en el laboratorio y posteriormente hubiéramos medido el espectro transmitido por las distintas gafas, nuestros resultados no podrían ser extrapolados a otros dispositivos y por tanto estarían limitados a los que nosotros empleamos en nuestro estudio. Por ello creemos que el enfoque metodológico que hemos escogido, basado en la determinación de la transmitancia de las lentes, permite conocer como será el espectro que llega al ojo para cualquier dispositivo móvil del que se pueda obtener su espectro de emisión, bien porque se mida o bien porque se obtenga de alguna base de datos de confianza.

Por otra parte, no es de extrañar que la transmitancia de las lentes sea tan alta. Uno de los problemas a los que se enfrentan los fabricantes de lentes oftálmicas al desarrollar el filtro azul es no alterar la cromática. Y esto tienen un balance muy peligroso. Queremos filtrar el azul, pero no alterar la percepción de los colores. Eso no es posible, a no ser que casi no se filtre el azul. Y esto es lo que probablemente nos lleve a unos porcentajes de filtrado tan bajos. Sin embargo, todos aceptamos el cambiar el color de la pantalla cuando cambiamos la TCC, y lo vemos como normal, es algo que llevamos experimentando toda la vida con el ciclo de luz diario. Por lo que la modificación de la CTT se presenta como una alternativa aceptable que ofrece una mayor reducción de la exposición.

Por otra parte, es importante poner en contexto la exposición a la luz azul nociva. Comentábamos al principio que la principal fuente de luz azul es el sol. ¿Cuál sería la dosis de luz azul nociva que recibiríamos si estuviéramos en el exterior un día soleado de 10.00 a 11.00 horas de la mañana? La respuesta es 30900 $\mathrm{mJ} / \mathrm{cm}^{2}$ [20]. Si en vez de estar al sol, hubiéramos estado en la habitación jugando con el Ipad Pro a una TCC de $5000 \mathrm{~K}$ durante esa hora, la dosis que habríamos recibido sería de $23.8 \mathrm{~mJ} / \mathrm{cm}^{2}$ [21], más de mil veces más. Mil veces más, así que para alcanzar la misma dosis deberíamos estar 1000 horas más jugando a la 
Tablet que jugando al baloncesto de 10.00 a $11.00 \mathrm{~h}$ en la calle. Y quien dice jugando al baloncesto, dice caminando hacia el trabajo o hacia el cole.

Así mismo, no podemos olvidar, que la exposición a la luz azul es necesaria para regular el ritmo circadiano y que se debe recibir una buena dosis de luz azul por la mañana para activar el cronómetro [7]. Por tanto, hay que tener cuidado con filtrar la luz azul solo pensando en sus efectos negativos, puesto que a lo mejor puede ser más negativo limitar sus efectos positivos.

\section{Conclusiones}

Según los resultados obtenidos en el presente trabajo, podemos concluir que el uso de lentes con filtro azul para proteger al usuario de la luz azul nociva o de la luz azul melanópica emitida por los dispositivos electrónicos como móviles, tablets, o monitores, no es lo más idóneo. La transmitancia de las lentes medidas ronda el $90 \%$ en el rango de emisión de estos dispositivos, un 6\% menor que la transmitancia de una lente convencional sin filtro azul, de forma que la protección que ofrecen es mínima. Por otra parte, hemos visto que, con el simple hecho de modificar la TCC de las pantallas, y pasar de $5000 \mathrm{~K}$ a $2700 \mathrm{~K}$, se consigue un mayor efecto protector que empleando las lentes propuestas. De esta forma, de tener que hacer una valoración, nos atreveríamos a indicar que dichos filtros no son la solución más adecuada puesto que no proporcionan una disminución significativa de la radiación recibida por el ojo en el rango de la luz azul nociva o luz azul melanópica, siendo mejor opción disminuir la TCC y brillo del dispositivo.

\section{Acknowledgements}

Este trabajo se realizó gracias a las aportaciones del Ministerio de Economía y Competitividad FIS201677319-C2-1-R, la Conselleria de Educación de la Xunta de Galicia, ayudas a la consolidación de grupos de investigación ED431B2017/64. 